\title{
References
}

1 Laveneziana P, Garcia G, Joureau B, et al. Dynamic respiratory mechanics and exertional dyspnoea in pulmonary arterial hypertension. Eur Respir J 2013; 41: 578-587.

2 Richter MJ, Voswinckel R, Tiede H, et al. Dynamic hyperinflation during exercise in patients with precapillary pulmonary hypertension. Respir Med 2012; 106: 308-313.

3 Naeije R. Lung mechanics and exertional dyspnea in pulmonary arterial hypertension. Respiration 2014; 88: $16-17$.

4 Laviolette L, Laveneziana P. Dyspnoea: a multidimensional and multidisciplinary approach. Eur Respir J 2014; 43: $1750-1762$.

5 Hoeper MM, Bogaard HJ, Condliffe R, et al. Definitions and diagnosis of pulmonary hypertension. J Am Coll Cardiol 2013; 62: Suppl., D42-D50.

6 Pellegrino R, Viegi G, Brusasco V, et al. Interpretative strategies for lung function tests. Eur Respir J 2005; 26: 948-968.

7 Laveneziana P, Webb KA, Wadell K, et al. Does expiratory muscle activity influence dynamic hyperinflation and exertional dyspnea in COPD? Respir Physiol Neurobiol 2014; 199: 24-33.

8 Laveneziana P, Webb KA, Ora J, et al. Evolution of dyspnea during exercise in chronic obstructive pulmonary disease: impact of critical volume constraints. Am J Respir Crit Care Med 2011; 184: 1367-1373.

9 O'Donnell DE, Revill SM, Webb KA. Dynamic hyperinflation and exercise intolerance in chronic obstructive pulmonary disease. Am J Respir Crit Care Med 2001; 164: 770-777.

10 ATS/ERS Statement on respiratory muscle testing. Am J Respir Crit Care Med 2002; 166: 518-624.

11 Younes M, Kivinen G. Respiratory mechanics and breathing pattern during and following maximal exercise. J Appl Physiol Respir Environ Exerc Physiol 1984; 57: 1773-1782.

12 Yan S, Kaminski D, Sliwinski P. Reliability of inspiratory capacity for estimating end-expiratory lung volume changes during exercise in patients with chronic obstructive pulmonary disease. Am J Respir Crit Care Med 1997; 156: 55-59.

13 Agostoni P, Pellegrino R, Conca C, et al. Exercise hyperpnea in chronic heart failure: relationships to lung stiffness and expiratory flow limitation. J Appl Physiol (1985) 2002; 92: 1409-1416.

14 Meyer FJ, Lossnitzer D, Kristen AV, et al. Respiratory muscle dysfunction in idiopathic pulmonary arterial hypertension. Eur Respir J 2005; 25: 125-130.

15 Kabitz HJ, Schwoerer A, Bremer HC, et al. Impairment of respiratory muscle function in pulmonary hypertension. Clin Sci (Lond) 2008; 114: 165-171.

16 de Man FS, van Hees HW, Handoko ML, et al. Diaphragm muscle fiber weakness in pulmonary hypertension. Am J Respir Crit Care Med 2011; 183: 1411-1418.

\section{Long-term mortality assessment of multidrug-resistant tuberculosis patients treated with delamanid}

\section{To the Editor:}

Multidrug-resistant tuberculosis (MDR-TB) is a serious obstacle to TB control [1]. The disproportionately negative outcomes among patients with drug resistance reflect a strong global need to develop new anti-TB drugs $[2,3]$. Delamanid is a novel anti-TB agent that has recently been approved for the management of MDR-TB patients [4]. Treatment of MDR-TB patients with delamanid in combination with an optimised background regimen for 2 months significantly improved 2-month sputum culture conversion (SCC) by $\sim 50 \%$, in comparison to treatment with placebo plus an optimised background regimen [5]. Additionally, compared to $\leqslant 2$ months of treatment, $\geqslant 6$ months of treatment with delamanid plus an optimised background regimen was associated with higher favourable treatment outcomes (55.0\% versus $74.5 \%)$ and significantly lower mortality $(8.3 \%$ versus $1.0 \%, \mathrm{p}<0.001)$ [6]. While early SCC is recognised as a biomarker in the development of anti-TB drugs [7-9], the impact of early SCC on long-term mortality in MDR-TB patients has only been assessed in retrospective cohort analyses [10-13]. Using updated prospective data from the delamanid development programme, we assessed the association between 2-month SCC and mortality in MDR-TB patients and expanded a previous analysis on the impact of long-term treatment with delamanid on mortality.

The clinical development programme for delamanid involved three consecutive trials: 1) a randomised placebo-controlled trial of 481 patients (Trial 204) for 3 months (2 months delamanid treatment plus 


\begin{tabular}{|c|c|c|c|}
\hline & Patients & Mortality & OR $(95 \% \mathrm{CI})$ \\
\hline \multicolumn{4}{|c|}{ 2-month sputum culture conversion } \\
\hline Yes & 192 & $6(3.1)$ & $0.25(0.10-0.61)^{\#}$ \\
\hline No & 272 & $31(11.4)$ & 1.00 \\
\hline \multicolumn{4}{|c|}{ Delamanid treatment duration } \\
\hline$\geqslant 6$ months & 205 & $6(2.9)$ & $0.22(0.09-0.54)^{\pi}$ \\
\hline$\leqslant 2$ months & 259 & $31(12.0)$ & 1.00 \\
\hline Total & 464 & 37 & \\
\hline
\end{tabular}

Data are presented as $n$ or $n(\%)$, unless otherwise stated. ${ }^{\#}: p=0.002 ;{ }^{~ ๆ}: p=0.001$.

1 month blinded follow-up); 2) an open-label treatment trial of 213 patients (Trial 208) with delamanid for 6 months (for any patient participating in Trial 204); and 3) a follow-up study of treatment outcomes of 421 patients (Trial 116) 24 months post-randomisation in Trial 204 [14]. Patients also received an optimised background regimen under directly observed treatment (as per World Health Organization guidelines [15]) throughout Trial 204 and Trial 208 and for the duration of the treatment period, generally 18-24 months.

The definition used for 2-month SCC in Trial 204 was five or more consecutive weekly cultures that were negative for growth (measured on liquid broth medium using the mycobacterial growth indicator tube automated system (Becton Dickinson, Franklin Lakes, NJ, USA)) of Mycobacterium tuberculosis, and no subsequent positive cultures during the follow-up period. Mortality during MDR-TB treatment among patients was determined at 24 months post-randomisation in Trial 204 based on information available from Trial 116 [13].

The primary outcome of Trial 204, 2-month SCC, was assessed in all 481 patients. Vital status was assessed and updated at $\geqslant 24$ months in 464 (96.5\%) patients, including 43 patients who did not participate in previously reported results of Trial 116. Paralleling a previous analysis, long-term treatment with delamanid was indicated as $\geqslant 6$ months, defined by assignment to delamanid or placebo in Trial 204 and participation in Trial 208, and short-term treatment with delamanid was indicated as $\leqslant 2$ months, defined by assignment to delamanid or placebo in Trial 204 and no participation in Trial 208 [6]. Mortality was also assessed by fitting a logistic regression model with vital status as response variables, and 2-month SCC status and delamanid treatment duration as factors.

2-month SCC was significantly $(\mathrm{p}=0.002)$ associated with a lower likelihood of mortality (OR $0.25,95 \%$ CI $0.10-0.61$ ). Among patients with 2-month SCC, 3.1\% (six out of 192) died compared to $11.4 \%$ (31 out of 272) who did not achieve 2-month SCC (table 1). Additionally, mortality was lower among patients within each of the original Trial 204 treatment groups for patients with 2-month SCC versus patients without 2-month SCC: $3.5 \%$ (five out of 142 ) versus $10.0 \%$ (17 out of 170 ) in the delamanid group and $2.0 \%$ (one out of 50 ) versus $13.7 \%$ (14 out of 102) in the placebo group.

Patients with long-term treatment with delamanid also had a significantly $(\mathrm{p}=0.001)$ lower likelihood of mortality (OR 0.22, 95\% CI 0.09-0.54). 2.9\% (six out of 205) of patients receiving delamanid for $\geqslant 6$ months died in comparison to $12.0 \%$ (31 out of 259) of patients who received delamanid for $\leqslant 2$ months (table 1). In the original Trial 204 randomised population, mortality was lower at $7.1 \%$ (22 out of 312) in patients assigned to delamanid compared to $9.9 \%$ (15 out of 152) for patients assigned to placebo. Of note, mortality among patients receiving delamanid for $\geqslant 6$ months was $2.9 \%$ (six out of 205) compared to $14.5 \%$ (12 out of 83 ) in those patients not receiving any treatment with delamanid.

The potential association between delamanid treatment duration, 2-month SCC and reduced mortality was also assessed. Among the 192 patients with 2-month SCC in Trial 204, mortality was 0\% among the 95 patients who then participated in Trial 208 versus 6.2\% among the 97 who did not participate in Trial 208. Likewise, among the 272 patients without 2-month SCC in Trial 204, mortality was lower at 5.5\% among the 110 patients who participated in Trial 208 versus $15.4 \%$ among the 162 patients who did not participate in Trial 208. Mortality was also consistently lower within each of the original Trial 204 treatment groups for patients with 2-month SCC who then participated in Trial 208 versus those who did not participate in Trial 208: 0\% (none out of 70) versus 6.9\% (five out of 72) in the delamanid group and $0 \%$ (none out of 25 ) versus $4.0 \%$ (one out of 25 ) in the placebo group. Finally, taken together and independent of Trial 204 treatment assignment, patients participating in Trial 208 had a lower mortality of $2.9 \%$ (six out of 205) compared to $12.0 \%$ (31 out of 59) in patients not participating in Trial 208. 
To assess variation among patients who enrolled in Trial 208 versus those who did not, baseline characteristics (cavitation, degree of drug resistance and previous treatment history) generally associated with negative treatment outcomes in MDR-TB patients were assessed for the 44.3\% (213 out of 481) of Trial 204 patients who participated in Trial 208 versus the 55.7\% (268 out of 481) of Trial 204 patients not entering Trial 208. Bilateral cavitation was present in 29.6\% (63 out of 213) of Trial 208 patients compared to $19.4 \%$ (52 out of 268) not entering Trial 208. Extensively drug-resistant patients and pre-extensively drug-resistant patients were in greater proportion among Trial 208 participants (81 (38.0\%) out of 213) compared to nonparticipants (54 (20.1\%) out of 268). Additionally, more patients in Trial 208 were previously treated with second- and third-line anti-TB drugs compared to patients not entering Trial 208 (109 (51.2\%) out of 213 versus 77 (28.7\%) out of 268).

This analysis is limited by at least three key issues: 1) the variable time-period between Trial 204 and Trial 208 allowing for continued treatment with an optimised background regimen in the absence of delamanid; 2) the open-label design of Trial 208; and 3) the lack of autopsy data to distinguish all-cause mortality from TB-related mortality. However, lower mortality trends with long-term treatment with delamanid were still observed despite more severely diseased patients participating in Trial 208 compared to nonparticipants.

These results support important conclusions regarding 2-month SCC and the treatment of MDR-TB patients with delamanid. First, 2-month SCC can be considered an important surrogate end-point in the treatment of MDR-TB patients, given the 3.7-fold lower mortality proportions among patients achieving 2-month SCC. Secondly, the expanded vital status results confirmed previously reported results [6] that the greatest reduction in mortality (a four-fold reduction) occurred among patients treated with delamanid for $\geqslant 6$ months versus those treated for $\leqslant 2$ months. Finally, delamanid may represent an important therapeutic option in the treatment of MDR-TB patients, as supported by the five-fold reduction in mortality among patients receiving delamanid for $\geqslant 6$ months compared to patients not receiving any treatment with delamanid.

Rapid evaluation of anti-TB drugs will require novel methods including the use of surrogate end-points. Such evaluations should ultimately include parameters to assess long-term outcomes, including mortality.

MDR-TB patients treated with 6 months or more of delamanid had significantly lower likelihood of mortality http://ow.ly/HOv1d

Charles D. Wells ${ }^{1}$, Rajesh Gupta ${ }^{1}$, Norbert Hittel ${ }^{2}$ and Lawrence J. Geiter ${ }^{1}$

${ }^{1}$ Otsuka Pharmaceutical Development and Commercialization, Rockville, MD, USA. ${ }^{2}$ Otsuka Novel Products, Munich, Germany.

Correspondence: Charles D. Wells, Otsuka Pharmaceutical Development and Commercialization, 2440 Research Boulevard, Rockville, MD 20850, USA. E-mail: charles.wells@otsuka-us.com

Received: Sept 252014 | Accepted after revision: Dec 192014 | First published online: Feb 192015

Conflict of interest: Disclosures can be found alongside the online version of this article at erj.ersjournals.com

\section{References}

1 Falzon D, Gandhi N, Migliori GB, et al. Resistance to fluoroquinolones and second-line injectable drugs: impact on multidrug-resistant TB outcomes. Eur Respir J 2013; 42: 156-168.

2 Migliori GB, Sotgiu G, Gandhi NR, et al. Drug resistance beyond extensively drug-resistant tuberculosis: individual patient data meta-analysis. Eur Respir J 2013; 42: 169-179.

3 Lienhardt C, Raviglione M, Spigelman M, et al. New drugs for the treatment of tuberculosis: needs, challenges, promise, and prospects for the future. J Infect Dis 2012; 205: Suppl. 2, S241-S249.

$4 \quad$ Ryan NJ, Lo JH. Delamanid: first global approval. Drugs 2014; 74: 1041-1045.

5 Gler MT, Skripconoka V, Sanchez-Garavito E, et al. Delamanid for multidrug-resistant pulmonary tuberculosis. N Engl J Med 2012; 366: 2151-2160.

6 Skripconoka V, Danilovits M, Pehme L, et al. Delamanid improves outcomes and reduces mortality for multidrug-resistant tuberculosis. Eur Respir J 2012; 41: 1393-1400.

7 Mitchison DA. Antimicrobial therapy of tuberculosis: justification for currently recommended treatment regimens. Semin Respir Crit Care Med 2004; 25: 307-315.

8 U.S. Department of Health and Human Services, Food and Drug Administration, Center for Drug Administration and Research. Guidance for Industry Pulmonary Tuberculosis: Developing Drugs for Treatment. Washington, DC, United States Health and Human Services, 2013.

9 Committee for Medical Products for Human Use. Addendum to the Note for Guidance on Evaluation of Medicinal Products Indicated for the Treatment of Bacterial Infections to Specifically Address Clinical Development of New Agents to Treat Disease Due to Mycobacterium Tuberculosis. London, European Medicines Agency, 2008.

10 Gammino VM, Taylor AB, Rich ML, et al. Bacteriologic monitoring of multidrug-resistant tuberculosis patients in five DOTS-Plus pilot projects. Int J Tuberc Lung Dis 2011; 15: 1315-1322. 
11 Holtz TH, Sternberg M, Kammerer S, et al. Time to sputum culture conversion in multidrug-resistant tuberculosis: predictors and relationship to treatment outcome. Ann Intern Med 2006; 144: 650-659.

12 Leimane V, Dravniece G, Riekstina V, et al. Treatment outcome of multidrug/extensively drug-resistant tuberculosis in Latvia, 2000-2004. Eur Respir J 2010; 36: 584-593.

13 Kurbatova EV, Taylor A, Gammino VM, et al. Predictors of poor outcomes among patients treated for multidrug-resistant tuberculosis at DOTS-plus projects. Tuberculosis (Edinb) 2012; 92: 397-403.

14 Diacon AH, von Groote-Bidlingmaier F, Donald PR. Delamanid, a new 6-nitro-2,3-dihydroimidazo[2,1-b] oxazole for the management of tuberculosis resistant to at least isoniazid and rifampicin. Expert Opin Orphan Drugs 2014; 2: 87-94.

15 Falzon D, Jaramillo E, Schünemann HJ, et al. WHO guidelines for the programmatic management of drug-resistant tuberculosis: 2011 update. Eur Respir J 2011; 38: 516-528.

\title{
Is 1-year follow-up adequate for adult tuberculosis contacts?
}

\author{
To the Editor:
}

To eliminate tuberculosis (TB) on a global scale, the identification and neutralisation of latently infected high-risk individuals is of paramount importance [1]. Active TB contacts are well documented with an increased risk for both latent TB infection (LTBI) and the development of active TB disease [2]. Contact investigation is an important and effective active case-finding strategy, but also requires abundant public health resources [3].

The current World Health Organization (WHO) recommendation for contact investigation suggests that follow-up screening should be considered particularly in the first year after exposure, such as after 6 or 12 months [4]. However, most of TB contacts studies have included both adults and children, or enrolled children only. It remains uncertain if the 1-year follow-up period is adequate for all TB contacts [5]. To optimise the contact investigation strategy for adult TB contacts, understanding the dynamic pattern of active TB development is important. Characterising the clinical features of adult TB contacts with a higher risk for active TB progression is helpful to prioritise medical resources more efficiently. It also enables researchers to select individuals who will benefit the most from LTBI treatment in resource-limited areas.

To elucidate the issue, we conducted a nationwide population-based cohort study using data retrieved from the National Health Insurance Research Database (NHIRD) in Taiwan between January 2000 and December 2011. TB contacts were identified by diagnostic codes (V01.1 in the International Classification of Diseases (ICD), 9th revision, and the clinical modification ICD-9-CM) in conjunction with chest plain film radiography and/or sputum acid-fast smear examination and/or tuberculin skin test (TST). We also matched each TB contact with four nonexposed subjects by age and sex, and presence of comorbidities on the same index date of diagnosis. The TB contacts cohort and the matched cohort were followed until the development of active TB disease, which was defined by compatible ICD-9-CM codes (010-018 in ICD-9-CM) [6, 7]. The diagnosis of TB was validated by the prescription of at least two anti-TB medications for more than 28 days. If no TB occurred, subjects were followed until death, or the end of the study period (December 2011). Incidence rates (per 100000 person-years) of active TB were analysed, and hazard ratios were calculated as compared with matched controls. For multivariate adjustment, the Cox proportional hazards model was used to compute hazard ratios (HRs) and 95\% confidence intervals. All statistical analyses were performed using SAS 9.2 software (SAS Institute Inc., Cary, NC, USA).

A total of 8659 TB contacts were identified during the study period. Their median age was 44 years old, $13.8 \%$ were $\geqslant 65$ years of age, and $44.2 \%$ of them were male. This cohort was matched with 34636 individuals without TB contact history in terms of age, sex and major comorbidities. The median (interquartile range (IQR)) follow-up times for TB contacts and their matched controls were 2.25 (1.06-3.69) years and 2.27 (1.07-3.70) years, respectively. Overall, $72 \mathrm{~TB}$ contacts and 67 individuals from the matched cohort were documented with active TB during the follow-up period, and the mean annual incidences of TB were 312.8 and 72.2 cases per 100000 person-years, respectively (adjusted HR 4.39, 95\% CI 3.15-6.12; p<0.001). The Kaplan-Meier analysis and annual incidences of active TB in TB contacts and control participants are shown 